\title{
Forecasting very short-term wind power generation using deep learning, optimization and data decomposition techniques
}

\author{
Md Alamgir Hossain ${ }^{1}$, Evan MacA. Gray ${ }^{1}$, Md Rabiul Islam ${ }^{2}$, Ripon K. Chakrabortty ${ }^{2}$, and Hemanshu R. Pota ${ }^{2}$
}

\begin{abstract}
Although accurate wind power prediction can improve the reliability, security and economic operation of a power system, the prediction task is complex due to the intermittent nature of wind speed its strong dependence on weather conditions. This paper proposes a novel framework, consisting of hybrid deep learning models, an optimization algorithm and a data decomposition technique, to improve the forecasting accuracy of ultra-short-term wind power generation. The data of the wind power generation collected from a real wind farm are preprocessed and decomposed using a variational mode decomposition (VMD). A deep-learning model (long short-term memory (LSTM) with dropout regularization) is designed to accurately predict the decomposed spectra. The hyper-parameters of the model are optimized by applying the grey-wolf optimization (GWO) algorithm to choose the best hyper-parameters. The combination of deep learning and optimization algorithm plays a key role in achieving better prediction accuracy. The effectiveness of the proposed framework is measured by applying it to two data sets, and the framework is compared with other forecasting models, such as a hybrid deep-learning and empirical wavelet transform (EWT) with LSTM. Comparison with experimental results demonstrates that the novel hybrid framework has the best prediction accuracy in forecasting ultra short-term wind power generation.
\end{abstract}

Index Terms - Very-short term prediction, wind power generation, VMD, data decomposition, deep learning, optimization algorithm, Boco Rock wind farm.

\section{INTRODUCTION}

The increasing integration of renewable energy sources, especially wind power generators, into power system networks has a great impact on the power system reliability and security due to the intermittent nature of power generation, leading to a higher mismatch in power supply and demand during scheduling and real-time operation [1]. In practice, this mismatch is balanced using either an energy storage system or an extra generating unit for operating the network in real time. This method raises many issues for managing the spinning reserve, such as higher operational and maintenance costs to provide backup power supply and environment pollution due to the disposal of these units [2]. These issues can be mitigated by enhancing the prediction accuracy of wind power generation, although forecasting wind power generation is complex due

\footnotetext{
*This work was supported by the Griffith University

${ }^{1}$ M. A. Hossain and E. M. Gray are with the Queensland Micro-and Nanotechnology Centre, Griffith University, Nathan, 4111, Australia, E-mail : mdalamgir.hossainegriffith.edu.au

${ }^{2}$ M. R. Islam, R. K. Chakrabortty and H. R. Pota are with the School of Engineering and IT, University of New South Wales-Canberra, ACT, 2612, Australia
}

to its heavy dependence on weather, leading to higher nonlinearity than other renewable energy sources, such as solar PV panels.

There are many publications in the literature reporting attempts to develop accurate forecasting models, including physical, statistical, neural-network (NN) and deep-learning model. The physical and statistical models have higher prediction errors arising from inexact mathematical models, learning capability and adaptability [3]. The NN model suffers from over- and under-fitting, slow convergence, long dependency and higher training times [4]. To overcome these deficiencies, the deep-learning approach has recently gained attention for developing forecasting models. In [5], a novel hybrid deeplearning model consisting of convolutional layers, gated recurrent unit (GRU) and $\mathrm{NN}$ was proposed to forecast very short-term wind power generation. The hyper-parameters of the model were tuned using a time-consuming grid search technique. A hybrid forecasting model of GRU and LSTM was proposed in [6] to accurately predict the wind power generation of Boco Rock wind farm in Australia. In [7], a wavelet data decomposition technique was used to forecast the short-term and long-term wind power generation using a convolutional NN (CNN) model. A deep learning model consisting of a convolutional neural network, gated recurrent units (GRU) and a fully connected neural network is proposed in [8] to improve the prediction accuracy of the very-short-term (5-min and 10-min) wind power generation. Harris Hawks Optimization algorithm is used to tune the hyper-parameters of the forecasting model. In [9], a GRU was employed to predict short-term wind power generation. An LSTM algorithm whose weights were trained using a gradient descent technique was developed in [10]. A forecasting model consisting of LSTM, principal component analysis and wavelet decomposition was presented in [11].

Models reported in the literature for forecasting wind power generation have the following shortcomings: (1) they are for short- or long-term prediction; (2) multiple forecasted variables are required, leading to higher error; (3) conversion from wind speed to wind power is required; and (4) system parameter tuning is often neglected. To this end, this paper proposes a novel forecasting framework consisting of a data decomposition technique, deep-learning and an optimization algorithm to improve the prediction accuracy of very shortterm wind power generation. The hyper-parameters of every deep learning model are optimized by the GWO algorithm. 
The rest of the paper is organized as follows. Section II presents the forecasting model proposed to accurately predict the wind power generation of the Boco Rock wind farm. In Section III, the GWO algorithm with problem formulation is employed to tune the hyper-parameters of the forecasting model. Simulation results for two data sets are presented in Section IV. Section V provides concluding remarks.

\section{Proposed Forecasting Model}

The description of each technique is provided as follows.

Variational mode decomposition (VMD): VMD decomposes the time-series data of wind power generation into a discrete number of sub-data (modes), which have a certain sparsity property while reproducing the original timeseries data [12]. Each mode, $u_{k}$, is assumed to be compact around a centre frequency, $\omega_{t}$, that is identified during decomposition. In this method, errors are equally distributed among modes by dynamically calculating relevant bands. The VMD, overcoming the shortcoming of the Empirical Mode Decomposition (EMD) and strict filter bank boundaries of the Empirical Wavelet Transform (EWT), is an effective method of decomposition to deal with noise and sampling. In VMD, predetermined $\mathrm{K}$ modes are obtained by solving a constrained variational optimization problem by means of the alternate direction method of multipliers (ADMM). The resulting constrained variational problem can be expressed as follows:

$\min \left\{u_{k}\right\},\left\{\omega_{k}\right\}\left\{\sum_{k=1}^{K}\left\|\partial_{t}\left[\left(\delta(t)+\frac{j}{\pi t}\right) * u_{k}(t)\right] e^{-j \omega_{k} t}\right\|_{2}^{2}\right\}$

such that $\sum_{k=1}^{K} u_{k}=f$

where $\left\{u_{k}\right\}=\left\{u_{1}, \ldots, u_{K}\right\}$ and $\left\{\omega_{k}\right\}=\left\{\omega_{1}, \ldots, \omega_{K}\right\}$ are the full set of modes and the central frequencies, respectively, and $\delta$ is the Dirac function.

The problem in Eq. (1) can be unconstrained using the Lagrangian multipliers and a quadratic penalty function as follows:

$$
\begin{aligned}
& \mathcal{L}\left(\left\{u_{k}\right\},\left\{\omega_{k}\right\}, \lambda\right) \\
& =\alpha\left\{\sum_{k=1}^{K}\left\|\partial_{t}\left[\left(\delta(t)+\frac{j}{\pi t}\right) * u_{k}(t)\right] e^{-j w_{k} t}\right\|_{2}^{2}\right\} \\
& \quad+\left\|f(t)-\sum_{k=1}^{K} u_{k}(t)\right\|_{2}^{2}+\left\langle\lambda(t), f(t)-\sum_{k=1}^{K} u_{k}(t)\right\rangle .
\end{aligned}
$$

By applying ADMM, Eq. (2) can be solved to obtain all the $K$ modes in the frequency area $\left(u_{k}\right)$ for each iteration $n$.

Long short-term memory (LSTM): The LSTM network is developed in such a way using neurons that store and transfer signals over a long period to (a) achieve permanent memories, (b) reduce the signal rate loss and (c) retain longterm dependencies [13]. The features are executed using a gate system to control the signal flow, i.e. the gradient flow, for overcoming the vanishing gradient issues. In this network, four gate mechanisms (input, forget, update and output) are utilized. The mathematical modelling of the LSTM can be represented as follows:

$$
\begin{aligned}
i_{t} & =\sigma\left(W_{i} \cdot\left[h_{t-1} \cdot x_{t}\right]+b_{i}\right) \\
\tilde{c_{t}} & =\tanh \left(W_{c} \cdot\left[h_{t-1} \cdot x_{t}\right]+b_{c}\right) \\
f_{t} & =\sigma\left(W_{f} \cdot\left[h_{t-1}, x_{t}\right]+b_{f}\right) \\
c_{t} & =f_{t} \odot c_{t-1}+i_{t} \odot \tilde{c_{t}} \\
o_{t} & =\sigma\left(W_{o} \cdot\left[h_{t-1}, x_{t}\right]+b_{o}\right) \\
h_{t} & =o_{t} \odot \tanh \left(c_{t}\right)
\end{aligned}
$$

where $W_{i}$ and $W_{c}$ are the weight matrices of the $i_{t}$ and $\tilde{c_{t}}$, respectively; and $b_{i}$ and $b_{c}$ are the bias terms of the input gate, $W_{f}$ is the weight matrix of the gate layer used for stored or deleted information, $\sigma$ is the sigmoidal function, $b_{f}$ is the bias term of the gate layer, $W_{o}$ and $b_{o}$ are the weight matrix of the output gate layer and the bias term of the output gate layer, respectively. The square brackets indicate addition of two vectors. $\odot$ is the Hadamard product of two states.

As the LSTM model may suffer from over-fitting, a dropout strategy is applied in the information transmission process at several moments. The mathematical representation for generating a set of random vectors ranging from 0 to 1 to delete random status can be written as follows:

$$
r \sim \operatorname{Bernoulli}(p)
$$

where $r$ is random vectors and $p$ is the dropout rate.

\section{Optimization Algorithm}

The grey-wolf optimization (GWO) algorithm developed by Mirjalili et al. in 2014 is inspired by the hunting behavior and the hierarchy structure of wolf packs in nature [14]. As the algorithm has some attractive characteristics, such as fast convergence, higher accuracy and easy implementation steps, it has been employed in many applications, such as feature selection, path planning, power point tracking, and energy scheduling [15]. In the wolf park $(\alpha, \beta, \delta$ and $\omega)$, the alpha wolf is the leader, and beta, delta and gamma are the second, third and fourth orders of subordinate in the decision making process, respectively. The mathematical modeling of grey wolves surround the prey and their behaviour can be expressed as follows:

$$
\begin{aligned}
& F=\left|G \cdot X_{p r}(t)-X(t)\right| \\
& X(t+1)=X_{p r}(t)-D \cdot F
\end{aligned}
$$

where $X_{p r}$ and $\mathrm{X}$ are the prey position and grey wolf position, respectively, and $t$ indicates iteration number. $D$ and $G$ are coefficients that can be represented as follows:

$$
\begin{aligned}
& D=2 a \cdot r_{1}-a \\
& G=2 . r_{2}
\end{aligned}
$$

where $r_{1}$ and $r_{2}$ are random numbers [0 - 1] and the component $a$ linearly decreases from 2 to 0 over the iteration. The best solutions of the three parameters $(\alpha, \beta$ and $\delta)$ are saved during the optimization process, then the positions of $\omega$ wolves 


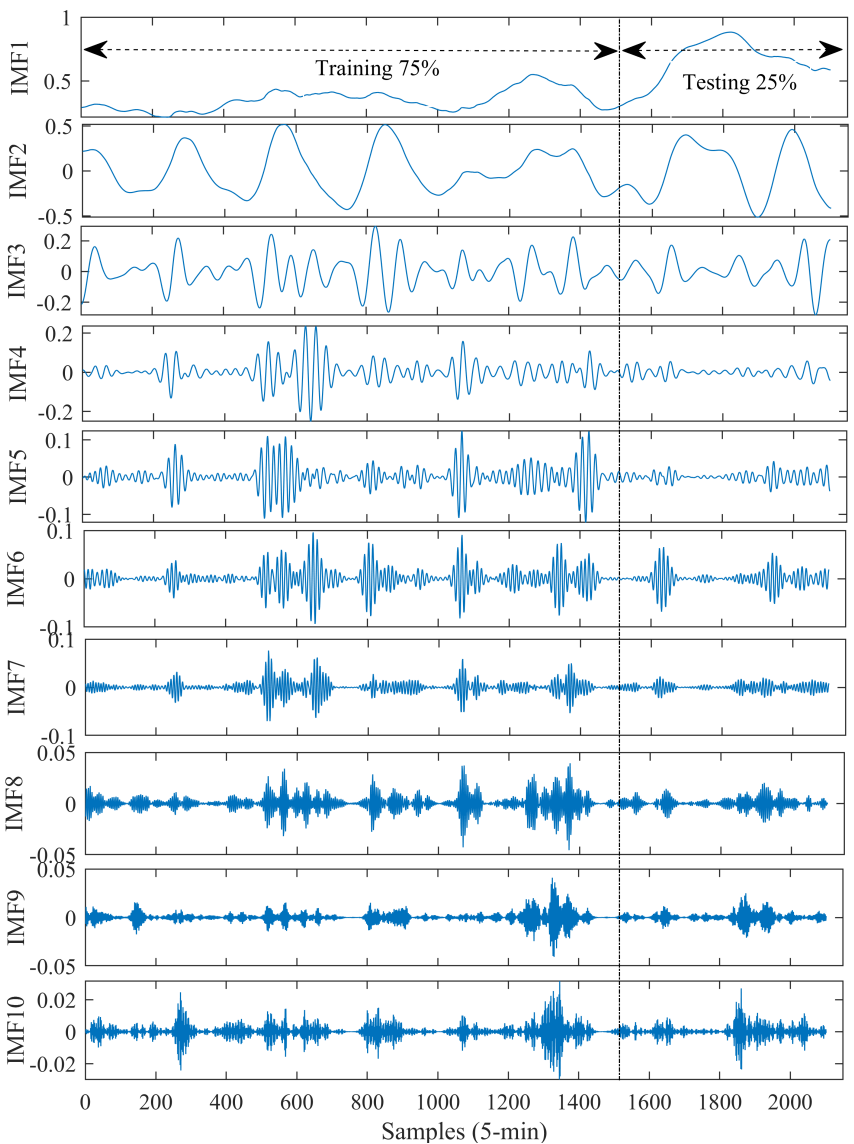

Fig. 1: Training and testing decomposed data set.

are updated. The process can be mathematically represented as follows:

$$
\begin{aligned}
& F_{\alpha}=\left|G_{1} \cdot X_{\alpha}(t)-X(t)\right| \\
& F_{\beta}=\left|G_{2} \cdot X_{\beta}(t)-X(t)\right| \\
& D_{\delta}=\left|G_{3} \cdot X_{\delta}(t)-X(t)\right|
\end{aligned}
$$

Depending on the position of alpha, beta and delta, the prey position can be measured as follows:

$$
\begin{aligned}
& X_{1}=\left|X_{\alpha}-D_{1} \cdot F_{\alpha}\right| \\
& X_{2}=\left|X_{\beta}-D_{2} \cdot F_{\beta}\right| \\
& X_{3}=\left|X_{\delta}-D_{3} \cdot F_{\delta}\right| \\
& X(t+1)=\frac{X_{1}+X_{2}+X_{3}}{3}
\end{aligned}
$$

where $X_{\alpha}, X_{\beta}$ and $X_{\delta}$ are the position of alpha, beta and delta, respectively. The exploration and exploitation of search agents depend on the parameter value of $D$, i.e., if $D \geq 1$, then exploration, otherwise, exploitation.

Problem formulation: To obtain the best combinations of hyper-parameters of deep learning, the GWO is used to minimize the Huber loss function through training the deep learning model. The model can be expressed as follows:

$$
\min D L(\widehat{H}, \widehat{W}, S)
$$

s.t.

$$
i_{D} \leq i_{\max }
$$

where $D L$ indicates the deep learning model, $\widehat{H}$ refers to a vector of hyper-parameters, $\widehat{W}$ is the vector of weights, and $S$ is the data set. $i_{D}$ indicates the number of internal iterations that will be less than the user-defined maximum number of iteration $\left(i_{\max }\right)$.

Huber loss function: The Huber loss function that provides a balanced response to outliers is used to update the weight of the deep learning [5]. The function, a combination of both Mean Square Error (MSE) and Mean Absolute Error (MAE), is given as follows:

$$
L_{\delta}(y, \hat{y})= \begin{cases}\frac{1}{2}(y-\hat{y})^{2} & \text { if }|y-\hat{y}| \leq \delta \\ \delta|y-\hat{y}|-\frac{1}{2} \delta^{2} & \text { otherwise }\end{cases}
$$

where $\delta$ is a hyperparameter that is a positive real number to regulate the transition between MSE and MAE.

Framework: The experimental procedure is described as follows: 1) the time-series data of wind power generation are decomposed to several sub-layers (viz. 10) by the VMD technique; (2) the sub-layers are divided into training and testing sets; (3) LSTM models with dropout regulation are constructed by using a training set to forecast wind power generation and the built models are employed to predict a testing set; and (4) the forecasting results of sub-layers are added to obtain the accurate prediction of wind power generation.

The deep-learning model is tuned using the GWO algorithm as follows: (1) set the parameter values, such as the population size (50) and the maximum generations (100); (2) define the search space of hyper-parameters; (3) Train the deep-learning model whose weights are updated using the Huber loss function; (4) evaluate the model with the performance matrices of the MAE and RMSE; (5) commence the optimization process with a large search space and reduce the search space for good performance, otherwise explore new search spaces; and (6) return the best combination of parameters.

\section{Simulation Results}

In this section, the performance of the proposed framework, consisting of data decomposition, deep-learning model and optimization algorithm, is examined to predict 5-min wind power generation of the Boco Rock wind farm. Using the GWO algorithm, the hyper-parameters of the deep learning model were selected as described in Section III. All the coding was implemented in Python 3.7 software using the Keras API integrated with TensorFlow. The very short-term data of wind power generation were collected and pre-processed for use in the deep-learning model. In total 2,102 data points were used to train and test the model to predict the wind power of 526 data points, i.e the data set was divided into $75 \%$ for training and $25 \%$ for testing the model. All the data were normalized before data decomposition for better prediction accuracy and returned the value in original form after prediction. 


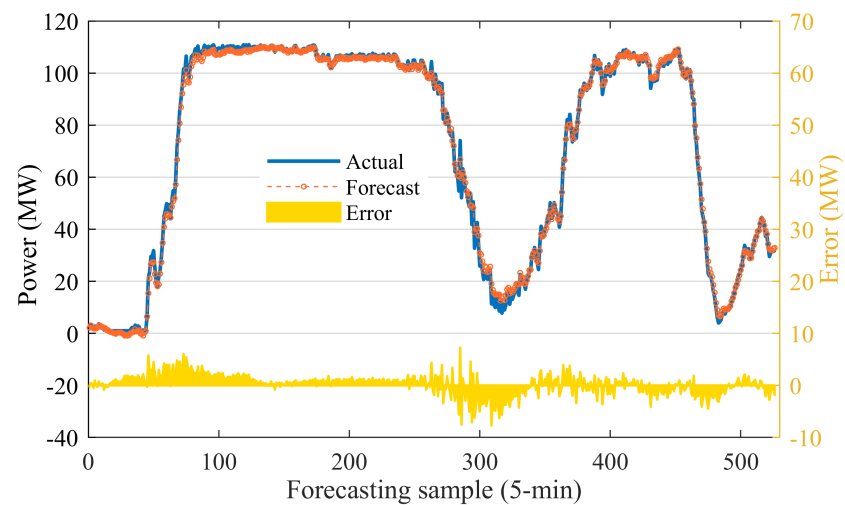

Fig. 2: Forecasting wind power generation for data set 1 .

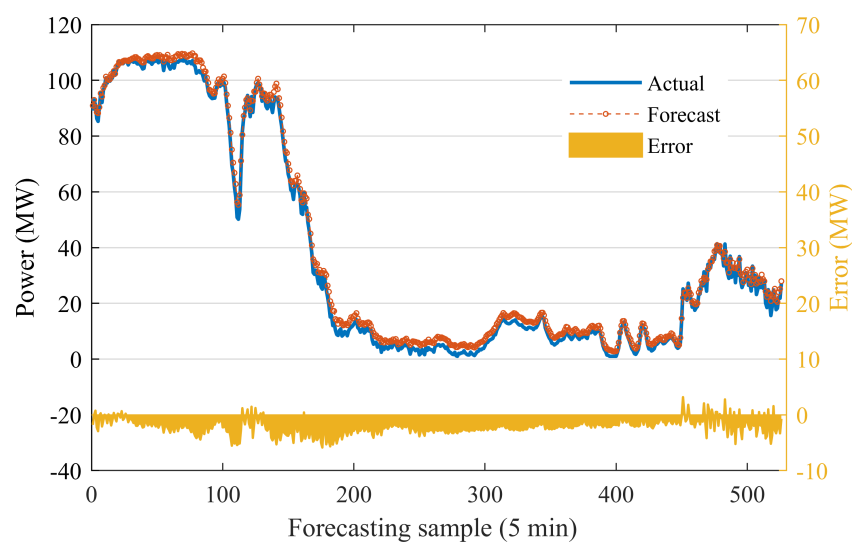

Fig. 3: Forecasting wind power generation for data set 2.

TABLE I: Comparison of the forecasting models for data set 1.

\begin{tabular}{cccccc}
\hline MAE & Best & Median & Mean & Worst & Std. \\
\hline Proposed & 1.40 & 1.64 & 1.65 & 1.94 & 0.1998 \\
EWT-LSTM & 3.75 & 4.01 & 4.04 & 4.50 & 0.2869 \\
CNN-GRU-NN & 2.43 & 2.84 & 2.85 & 3.35 & 0.4084 \\
\hline RMSE & & & & & \\
\hline Proposed & 1.87 & 2.09 & 2.12 & 2.54 & 0.2594 \\
EWT-LSTM & 4.52 & 4.82 & 4.88 & 5.39 & 0.3402 \\
CNN-GRU-NN & 3.80 & 4.04 & 4.06 & 4.34 & 0.2187 \\
\hline
\end{tabular}

TABLE II: Comparison of the forecasting models for data set 2.

\begin{tabular}{cccccc}
\hline MAE & Best & Median & Mean & Worst & Std. \\
\hline Proposed & 1.77 & 2.19 & 2.15 & 2.41 & 0.2356 \\
EWT-LSTM & 2.89 & 3.67 & 3.56 & 4.21 & 0.5081 \\
CNN-LSTM-NN & 1.94 & 2.24 & 2.23 & 2.61 & 0.2424 \\
\hline RMSE & & & & & \\
\hline Proposed & 2.07 & 2.50 & 2.49 & 2.86 & 0.2856 \\
EWT-LSTM & 3.89 & 4.78 & 4.64 & 5.34 & 0.5614 \\
CNN-GRU-NN & 2.69 & 2.81 & 2.82 & 3.05 & 0.1455 \\
\hline
\end{tabular}

\section{A. Data set 1: forecasting power generation}

The first data set of the 5-min sample was used to tune the hyper-parameters of the forecasting model using the GWO described in Section III. The GWO was also used to properly choose the hyper-parameters of the other forecasting models. The forecasting model was tuned on two layers of LSTM networks with a dropout rate at the middle, followed by a NN layer. From the outcomes of the optimization algorithm, the number of neurons was taken as 120 (ranging, 20-200), the dropout $0.4(0.1-0.8)$, an optimization algorithm for weight update 'Adam' (Adagrad, RMSprop and Adam), batch size 32 (16-128) and input window size 10 (3-50).

Fig. 2 shows the outcome of the proposed forecasting model for data set 1 . The forecasting outcome is closely following the actual power generation output of the Boco Rock wind farm. The majority of the error in the forecasted data remains less than $5 \mathrm{MW}$. The higher errors are observed when there are some transitions from low to high or vice-versa as this phase is always tricky to predict closely due to their nature. To calculate errors, mean absolute error (MAE) and root mean square error (RMSE) are used as performance indices. In the proposed model, the MAE is 1.56 and RMSE is 1.94, indicating lower error in forecasting the wind power generation of the Boco Rock wind farm.

\section{B. Data set 2: forecasting power generation}

In this section, the tuned prediction model is used to predict another data set of the wind farm with the same training and testing samples as described in the previous section. The forecasting results of the testing data set are demonstrated in Fig. 3. The actual wind power generation of the farm is again closely followed by the predicted power generation. In this data set, the model often overestimates the power generation, although the errors are mostly within $5 \mathrm{MW}$. Once again, higher errors occur during transitions. The performance indices for MAE and RMSE are 1.77 and 2.07, respectively.

\section{Comparative analysis}

This section is dedicated to comparing the forecasting outcome with other prediction methods. In this study, we compare the forecasting outcome with the Empirical Wavelet Transform (EWT) data decomposition technique [16] and a newly proposed hybrid deep learning model [5]. The parameters of the EWT are tuned using the grid search technique to obtain the best combination. In the EWT, we have chosen fifteen lengthFilter, fifteen sigmaFilter, and Gaussian in 'reg' with 8 data decomposition modes. As training deep learning, especially for data decomposition techniques, is a very timeconsuming process, we have run only 5 times to obtain the forecasting outcomes statistically that also demonstrate the stability of the proposed model. The outcomes of the forecasting model for data set 1 are demonstrated in Table I. Among the comparative results, the highest forecasting error is obtained in the EWT-LSTM model followed by the CNN-GRU-NN for both the performance (MAE and RMSE) indices. The lowest prediction errors are recorded in the proposed model 
followed by the CNN-GRU-NN one. From the median value, it can be concluded that the proposed model demonstrates better forecasting accuracy (around 59\% in MAE and 57\% in RMSE) as compared to the EWT-LSTM forecasting model. For data set 2, Table II demonstrates the outcome of the prediction models. The highest median prediction errors are observed in the EWT-LSTM model followed by the CNNLSTM-NN. The best forecasting result is again obtained by the proposed forecasting model. From the above analysis, it can be concluded that the proposed forecasting model has higher accuracy than other methods.

\section{CONCLUSiON}

This paper proposes a framework to forecast ultra shortterm wind power generation of the Boco Rock wind farm. The framework consists of the data decomposition technique, deeplearning models and an optimization algorithm. The LSTM model has proven its ability to retain the long dependency of input and output data while the dropout regularization has made the model robust against over-fitting. The performance of the proposed model with the data decomposition technique is further improved by employing the GWO algorithm to tune the hyper-parameters of the model. To demonstrate the effectiveness of the proposed model, a comparative analysis with other models, such as EWT-LSTM and CNN-LSTMNN, was carried out. From the analysis of two data sets, the proposed model exhibits performance superior to other advanced forecasting models for both data sets.

Several case studies with various types of forecasting methods will be performed to further evaluate the effectiveness of the proposed framework.

\section{REFERENCES}

[1] M. A. Hossain, H. R. Pota, M. J. Hossain, F. Blaabjerg, Evolution of microgrids with converter-interfaced generations: Challenges and opportunities, International Journal of Electrical Power \& Energy Systems 109 (2019) $160-186$.

[2] S. A. Khan, M. R. Islam, Y. Guo, J. Zhu, A new isolated multi-port converter with multi-directional power flow capabilities for smart electric vehicle charging stations, IEEE Transactions on Applied Superconductivity 29 (2) (2019) 1-4.

[3] N. Chen, Z. Qian, I. T. Nabney, X. Meng, Wind power forecasts using gaussian processes and numerical weather prediction, IEEE Transactions on Power Systems 29 (2) (2013) 656-665.

[4] J. Wang, W. Yang, P. Du, T. Niu, A novel hybrid forecasting system of wind speed based on a newly developed multi-objective sine cosine algorithm, Energy Conversion and Management 163 (2018) 134-150.

[5] M. A. Hossain, R. K. Chakrabortty, S. Elsawah, M. J. Ryan, Very shortterm forecasting of wind power generation using hybrid deep learning model, Journal of Cleaner Production 296 (2021) 126564.

[6] M. A. Hossain, R. K. Chakrabortty, S. Elsawah, M. J. Ryan, Hybrid deep learning model for ultra-short-term wind power forecasting, in: 2020 IEEE International Conference on Applied Superconductivity and Electromagnetic Devices (ASEMD), IEEE, 2020, pp. 1-2.

[7] O. Abedinia, M. Bagheri, M. S. Naderi, N. Ghadimi, A new combinatory approach for wind power forecasting, IEEE Systems Journal 14 (3) (2020) 4614-4625.

[8] M. A. Hossain, R. K. Chakrabortty, S. Elsawah, E. M. A. Gray, M. J. Ryan, Predicting wind power generation using hybrid deep learning with optimization, IEEE Transactions on Applied Superconductivity (2021).

[9] Z. Niu, Z. Yu, W. Tang, Q. Wu, M. Reformat, Wind power forecasting using attention-based gated recurrent unit network, Energy 196 (2020) 117081 .
[10] C. Li, G. Tang, X. Xue, X. Chen, R. Wang, C. Zhang, The short-term interval prediction of wind power using the deep learning model with gradient descend optimization, Renewable Energy 155 (2020) 197-211.

[11] Y. Sun, P. Wang, S. Zhai, D. Hou, S. Wang, Y. Zhou, Ultra shortterm probability prediction of wind power based on 1 stm network and condition normal distribution, Wind Energy 23 (1) (2020) 63-76.

[12] K. Dragomiretskiy, D. Zosso, Variational mode decomposition, IEEE transactions on signal processing 62 (3) (2013) 531-544.

[13] S. Han, Y.-h. Qiao, J. Yan, Y.-q. Liu, L. Li, Z. Wang, Mid-to-long term wind and photovoltaic power generation prediction based on copula function and long short term memory network, Applied energy 239 (2019) 181-191.

[14] S. Mirjalili, S. M. Mirjalili, A. Lewis, Grey wolf optimizer, Advances in engineering software 69 (2014) 46-61.

[15] Y. Fu, H. Xiao, L. H. Lee, M. Huang, Stochastic optimization using grey wolf optimization with optimal computing budget allocation, Applied Soft Computing 103 (2021) 107154.

[16] J. Gilles, Empirical wavelet transform, IEEE transactions on signal processing 61 (16) (2013) 3999-4010. 